\title{
HACIA LAS INTERVENCIONES TECNOLÓGICAS Y SUS IMPLICANCIAS SOCIALES. SIMILITUDES ENTRE CHANDIGARH Y LIMA
}

\author{
TOWARDS TECHNOLOGICAL \\ INTERVENTIONS AND THEIR SOCIAL \\ IMPLICATIONS. SIMILARITIES \\ BETWEEN CHANDIGARH AND LIMA
}

\author{
ARQ. VANESSA ZADEL \\ Universidad de Lima \\ ARQ. NISHTHA KAUSHIK \\ Universidad de Panjab
}

Este artículo expone las posibilidades y el impacto social que las intervenciones tecnológicas tienen en nuestras ciudades. Las bases comunes de estas implicancias tecnológicas comprenden los campos; por ejemplo, soluciones IoT, inteligencia artificial, movilidad inteligente y soluciones integradas de gestión de residuos. Los fundamentos comunes del impacto social son una herramienta poderosa para la formación de comunidades inteligentes, que pueden demostrar equidad y accesibilidad. Por lo tanto, existe la necesidad de espacios para los creadores de la comunidad que puedan ayudar a otros no solo a mantenerse al día con la tecnología y usarla de manera eficiente, sino también para conectarlos con nuevas comunidades y adaptar diferentes soluciones a diversas condiciones fisicas.

ciudades del futuro, internet de las cosas, manufactura digital y robótica, inteligencia artificial, revolución digital

\author{
ARQ. MAHAVIR SINGH \\ FabLab Chandigarh \\ Recibido: 29 de agosto del 2019 \\ Aprobado: 3 de octubre del 2019
}

doi: 10.26439/limaq2020.n006.4823

This article presents the possibilities and social impact that technological interventions have in our cities. Common bases for these technological implications include, for example, the fields of IoT solutions, artificial intelligence, smart mobility and integrated waste management solutions. The common foundations of social impact are a powerful tool for the formation of intelligent communities, which can demonstrate equity and accessibility. Therefore, there is a need for spaces for community creators that can help others not only to keep up with technology and use it efficiently but also to connect them with new communities and adapt different solutions to various physical conditions.

future cities, internet of things, digital and robotic fabrication, artificial intelligence, digital revolution 


\section{INTRODUCCIÓN}

Actualmente, la tecnología nos permite formar un tipo de vínculo que sobrepasa los límites físicos y produce nuevas formas de relacionarse con otras personas y con el medio que habitamos: nos enteramos en tiempo real de lo que sucede del otro lado del mundo e incluso lo comentamos y analizamos con personas que no conocemos y probablemente nunca conoceremos; celebramos reuniones de trabajo entre oficinas sin movernos de la nuestra; charlamos con una persona que está en otro continente sin importar las diferencias horarias: se puede dejar un mensaje grabado (hoy los mensajes de voz son más frecuentes que los de texto); con horas de vuelo de drones aprendemos de las muchas otras especies que habitan con nosotros. Hacemos todo esto y más sin que nuestras discapacidades físicas, mentales o sensoriales sean un impedimento.

Este artículo es un aporte a la lista de las varias posibilidades de enlace que nos ofrece el uso de la tecnología: arquitectos de Chandigarh (India) y Lima que trabajan juntos e imaginan los cambios que trae consigo la tecnología, además de la forma de comunicarse, y reflexionan al respecto. Cambios que comenzarán simultáneamente y que nos sacarán de nuestra zona de confort social. Nuestra capacidad para adaptarnos depende de esta utopía.

Como arquitectos nos interesa reflexionar sobre cómo nos conectamos con nuestro entorno y cómo este irá configurándose a medida que avance la tecnología (cada vez más rápidamente). Dentro de poco, estas intervenciones llegarán también a Chandigarh y a Lima y les permitirán convertirse en ciudades inteligentes, con la certeza de que puedan relacionarse sin importar sus límites físicos.

Figura 1. Chandigarh

Fuente:

Google Earth

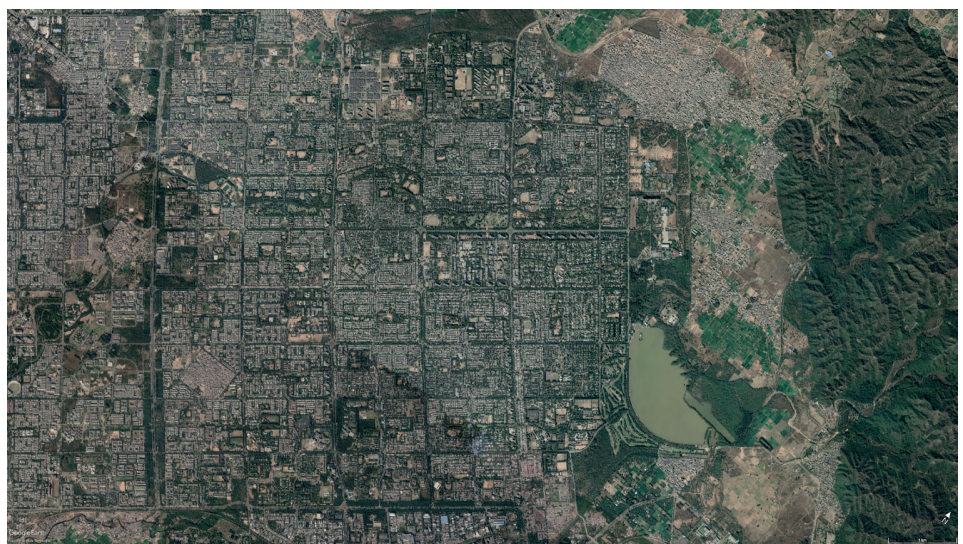

Chandigarh, la utopía urbana, está ubicada en las estribaciones del Siwalik, la cordillera asiática que corre paralela al Himalaya, al norte de India. Es la ciudad símbolo del nuevo inicio moderno de la India independiente y se la considera una ciudad ideal. Fue fundada en 1951, planificada por el famoso 
arquitecto franco-suizo Le Corbusier y construida en un momento determinante y excepcional en la historia de India, sobre un lugar vacío, respetando las normas de planificación alrededor de su topografía y una clara indiferencia a la preservación de un sitio arqueológico de la civilización de Harappa, que ahí existía. En la actualidad, una parte de la arquitectura de la civilización de Harappa permanece enterrada entre quince y veinte metros de profundidad.

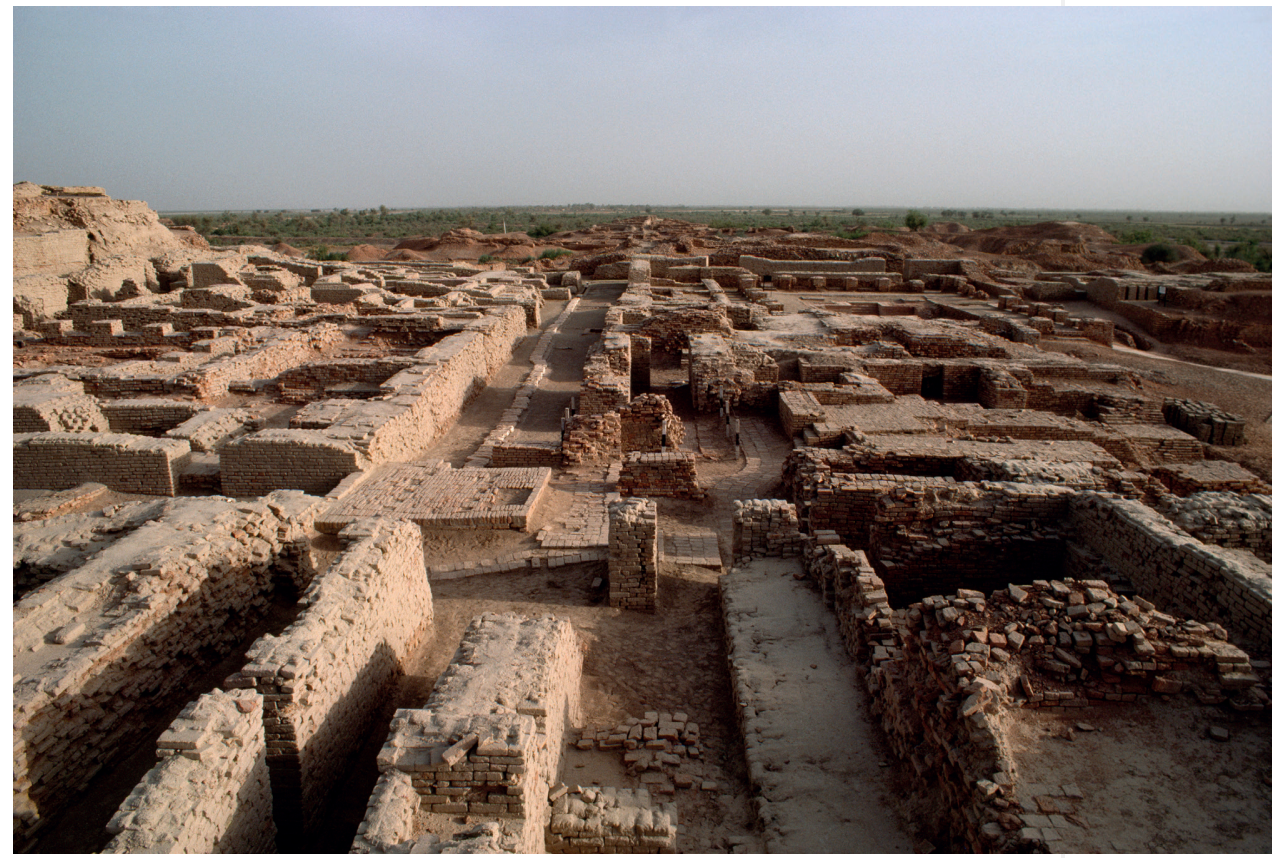

Chandigarh es conocida como una de las ciudades mejor planificadas. Está dividida en diferentes sectores, como la estructura de una cuadrícula. Cada sector tiene una franja verde que corre de norte a sur y los atraviesa una calle comercial que corre de este a oeste. Las calles están organizadas de manera decreciente y marcadas de V1 a V8: V1: carreteras principales que conectan una ciudad con otra, V2: calles urbanas, V3: calle vehicular que rodea un sector, V4: calle comercial de un sector, V5: vía colectora que serpentea un sector, V6: calle residencial, V7: senda peatonal, V8: senda para ciclistas. Cada sector está rodeado por calles $\mathrm{V} 2 \mathrm{o} \mathrm{V} 3$, sin construcciones que se abran a ellas, y ha sido diseñado para ser autosuficiente, con tiendas e instalaciones a las que se pueda ir caminando. Las sendas para ciclistas son muy importantes y su diseño y orientación les permiten estar bajo sombra la mayor parte del día.

Figura 2.

Restos arqueológicos de Harappa

\section{Fuente:}

https://blog. nationalgeogra phic.org/2015/04/ 11/exploring-civili zation-beyond-thewalls/

Desde sus primeros estudios en urbanismo, Le Corbusier ha identificado el automóvil como el factor central del urbanismo moderno. Su respuesta inicial a los automóviles y al rápido movimiento en las ciudades, básicamente estética y casi futurista para 1950, se convirtió en la solución teórica a los problemas del tráfico moderno: un sistema de circulación gradual 
que atraviesa continentes para llegar a la puerta de la casa. Las siete vías son al plano de la ciudad lo que los sistemas circulatorio, linfático y respiratorio, al cuerpo humano. Son sistemas lógicos, distintos uno del otro, no se confunden entre sí y mantienen la armonía. Debemos aprender de ellos para organizar el terreno donde vivimos. Las siete vías ya no son los instrumentos siniestros de la muerte, sino una disposición jerarquizada de calles que pueden controlar el flujo del tránsito moderno". (Prasad Sunand citado en Maria Thurockzy, 2014)

LIMA

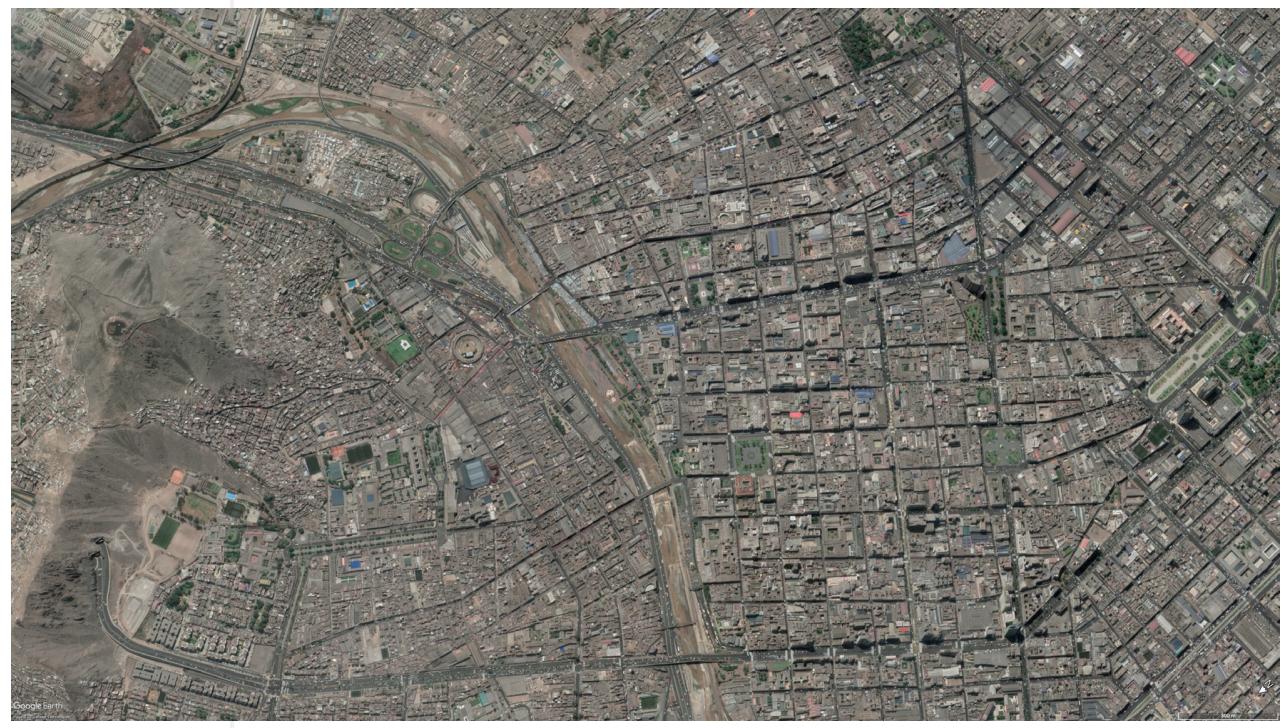

Figura 3

Lima

Fuente:

Google Earth

La ciudad de Lima, capital del Perú, fue fundada el 18 de enero de 1535 por el conquistador español Francisco Pizarro. El valle del río Rímac, el más extenso de la costa peruana, fue el lugar elegido para su establecimiento. La disposición de la ciudad fue de nueve calles dispuestas a lo largo y trece a lo ancho; un total de 117 manzanas, cada una dividida en cuatro solares. "La forma urbana deriva, en principio, de la traza en forma de damero o cuadrícula. Como elemento central y característico de todas las ciudades hispanoamericanas se situó la Plaza Mayor" (Bonilla Di Tolla, 2015, p. 151).

Los conquistadores españoles encontraron rutas y canales (acequias) incaicos que alteraron el plano de la ciudad. "Esto significó que las ciudades coloniales se desarrollaron en un contexto de asentamiento ya establecido, aislando y absorbiendo los antiguos restos del orden precolonial ecológico, social y espacial" (Davis, 2017, p. 19). Nuestros ancestros entendían el territorio en que habitaban y aprendieron a usar las diferentes condiciones a su favor. No en vano hasta nuestros días, a pesar de los diferentes fenómenos naturales, la 
destrucción provocada por el ser humano y el paso del tiempo, podemos ver rastros de las antiguas civilizaciones. Los restos arqueológicos, denominados huacas, son una suerte de testigos silenciosos del paso del tiempo y del crecimiento de la ciudad. Vivimos rodeados de una herencia cultural que, para muchos, está olvidada. Es común conocer limeños que nunca han visitado una huaca.

En Lima, más de 360 sitios arqueológicos prehispánicos están actualmente insertados en la trama urbana. Rodeados de muros y abandonados. Estos sitios no participan en la vida de la ciudad y son vistos por los ciudadanos como vacíos en el tejido urbano. (Crousse, 2017, pp. 9, 11)

La mayoría de estos lugares sagrados han sido mutilados. Por ejemplo, la huaca Pucllana ha sido atravesada por calles, como resultado de la expansión urbana, otras se han visto forzadas a compartir sus gruesos muros con nuevas construcciones y el crecimiento no planificado de la ciudad. Para José Canziani, Lima ha sufrido, en el siglo xx, la más grande destrucción de su legado cultural prehispánico cuando los identificó como los no lugares, "Espacios condenados al anonimato y a la pérdida de su identidad" (Canziani, 2013, p. 78).

¿Cómo se puede ser consciente de algo que no se sabe que existe? Probablemente la tecnología no solo nos brinda una nueva manera de percibir los sitios arqueológicos de nuestra ciudad, sino que también los hace "visibles" para todos.

Figura 4.

Huaca Pucllana, Miraflores

Fuente:

Google Earth

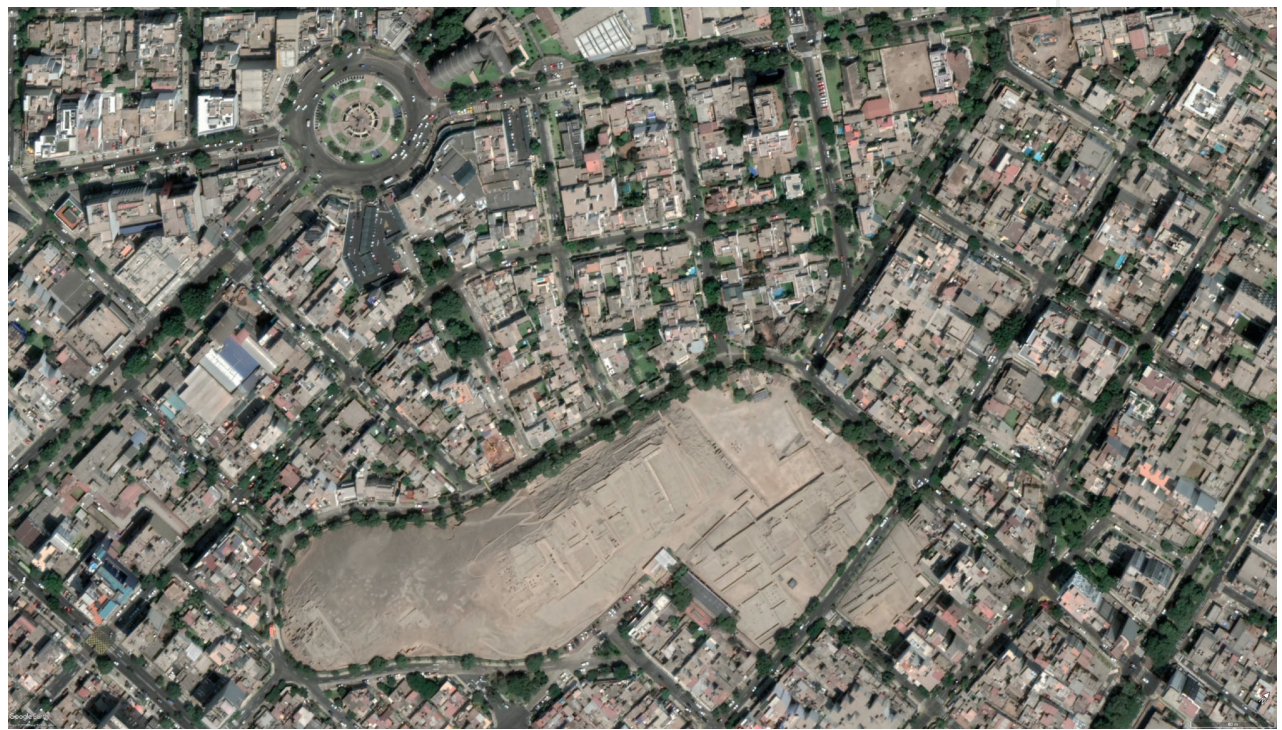




\section{PUNTOS DE ENCUENTRO}

Con más de dos tercios de nuestra población proyectada a vivir en áreas urbanas para el 2050, la carga en los servicios de la ciudad aumentará considerablemente. Los avances tecnológicos en la infraestructura urbana siempre han demostrado el impacto que tienen en la manera como los ciudadanos interactúan y viven en la sociedad. Además, con el próximo crecimiento en el campo de la inteligencia artificial y el internet de las cosas, las ciudades de todo el mundo se están volviendo inteligentes; incluso hasta permitiendo una infraestructura basada en reglas cívicas para empezar a adaptarse a la psicología humana. Repentinamente, la neurociencia y los laboratorios del comportamiento comenzarán a cobrar un nuevo significado.

La computación en la nube, la conectividad de banda ancha y los sensores sofisticados se juntan para cambiar la forma en que interactuamos, consumimos, nos trasladamos y le damos forma a un nuevo paisaje urbano. Con la adopción generalizada de una amplia variedad de tecnologías, productos y servicios digitales - en tiempo real y en la red- las personas, las compañías, los gobiernos e incluso las máquinas se mantienen conectados y se comunican entre sí, reuniendo, analizando e intercambiando grandes cantidades de información sobre todo tipo de actividades.

Cuando decimos "ciudad inteligente", se nos vienen a la mente todo tipo de servicios enfocados en los ciudadanos y en la conectividad con sus periferias. En el futuro, las bases comunes de las intervenciones sociales serán una herramienta poderosa para formar comunidades inteligentes que puedan demostrar equidad y accesibilidad mediante el uso de la tecnología. Una nueva definición de ciudad inteligente o ciudad tecnológica incluirá la necesidad de espacios para que los creadores de la comunidad puedan ayudar a la sociedad a ir a la par con la tecnología, a adaptarse a ella eficientemente, a conectarse con nuevas comunidades y a adaptar diferentes soluciones para distintas capacidades físicas.

La tercera revolución, también conocida como "revolución digital", ha estado vigente desde mediados del siglo xx y está relacionada con el uso de las tecnologías que buscan borrar los límites entre lo físico, lo digital y lo biológico.

Estamos a puertas de lo que Klaus Schwab, fundador del Foro Económico Mundial, denominó “la Cuarta Revolución Industrial”. Según Schwab, estamos en una etapa que estará marcada por los diferentes avances tecnológicos emergentes de campos, como la robótica, la inteligencia artificial, la cadena de bloques, la nanotecnología, la computación cuántica, la biotecnología, IoT, la impresión 3D y los vehículos autónomos. Entramos a una nueva utopía guiada por la tecnología, que no conoce límites ni condicionantes físicas.

En esta utopía Chandigarh y Lima, ciudades tan distantes entre sí, se acercan. El mundo ha comenzado a construir estructuras con una tecnología muy superior a la utilizada hasta ahora, y seguirá evolucionando. Es cada vez más 
frecuente oír del uso de brazos robóticos en la industria de la construcción. Los brazos robóticos, dirigidos por control numérico y que responden a diseños asistidos por computadora, aportan una nueva definición a la "construcción", en lo concerniente a tiempos, procesos y usos del material. Ni qué decir de la reducción en los costos de mano de obra y la amplitud de conocimientos relacionados con el diseño. No será raro ver cómo cada comunidad fabrica o, mejor dicho, "imprime" sus edificios, y esto será posible gracias a la suma de las tareas diversificadas.

El próximo año Dubái comenzará las pruebas del proyecto terminado de Mars Science City, una ciudad impresa en 3D con arena del desierto. Este proyecto es parte de la Misión Marte de los Emiratos Árabes Unidos, que busca colonizar Marte. Bjarke Ingels es el arquitecto que está a cargo de esta ciudad de $175000 \mathrm{~m}^{2}$. El término del proyecto está previsto para el 2020. Si ya estamos pensando en colonizar otros planetas, ¿qué nos impide pensar que en unos pocos años y gracias a las nuevas tecnologías las comunidades podrán fabricar los espacios que necesitan exactamente donde los necesitan?

Figura 5.

Mars Science City

\section{Fuente: http:/l} www.archtalent.com/es/ proyectos/ mars-sciencecity-8b4ab584e3ae-49f6-98aca53a69a569a4]

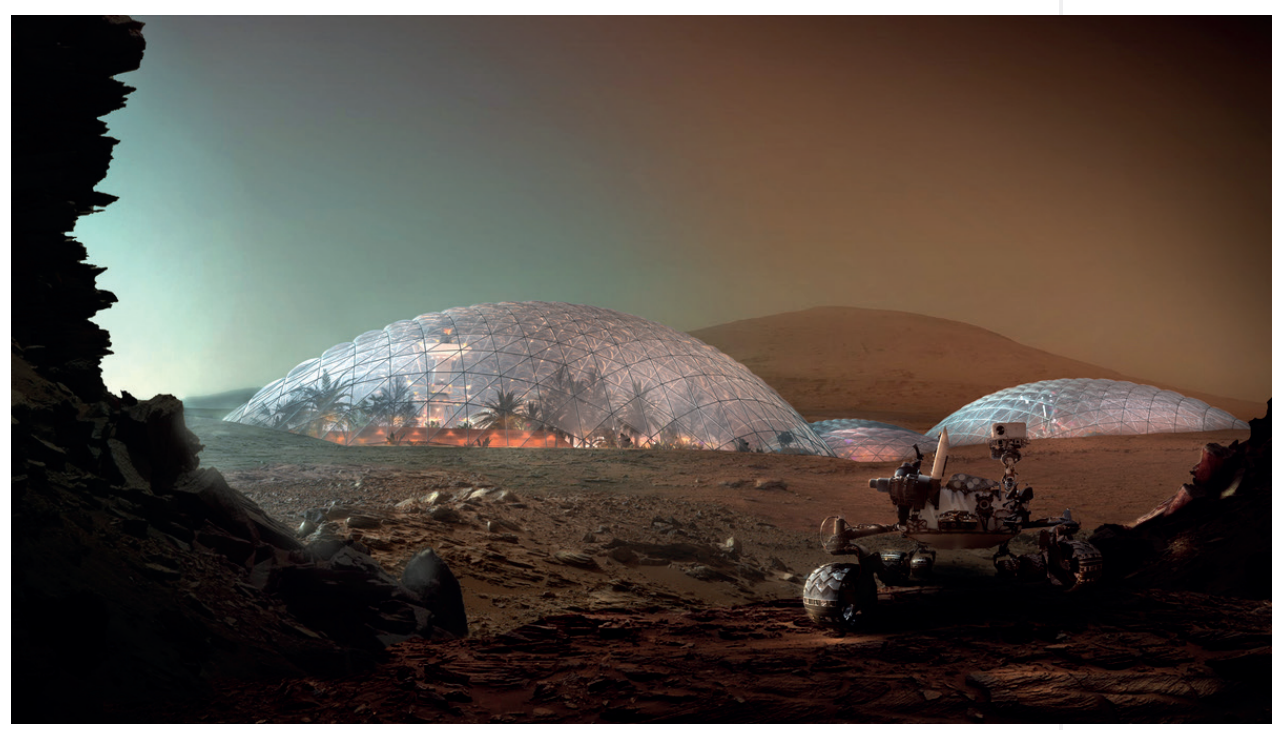

Hoy la proliferación de los laboratorios de fabricación digital pone a nuestro alcance un gran número de herramientas de alto costo que aún no son de uso frecuente en nuestros hogares y que, por ahora, no necesitamos adquirir. Esto abre las puertas al aprendizaje comunitario, un factor que mejorará las habilidades y las capacidades individuales. En pocos años, los niños y las niñas usarán impresoras PLA o ABS; será un nuevo nivel en la exploración de herramientas y nuevos materiales. Decimos: "Todos los niños y todas las niñas", sí, es lo ideal, pero, ¿qué es la utopía si no la suma de todos nuestros ideales? 
Figura 6 .

Jarrón cirratus.

Exposición "Meta

Utopia" en la

galería Zaha

Hadid

Fuente: http://

www.xtreee.eul

projects-cirra-

tus-vase/

La impresión 3D, además de traer consigo una nueva manera anticapitalista de fabricación del tipo "hágalo usted mismo", elimina las barreras físicas que una discapacidad puede imponer frente a nuestra exploración y aprendizaje. De pronto, una persona con impedimento visual puede tener piezas arqueológicas u objetos valiosos en sus manos y, al tocarlos, distinguir sus detalles y acceder a conocimientos que antes no tenía a su alcance. La tecnología posibilita nuevas formas de aprendizaje, de las cuales todos nos beneficiamos.

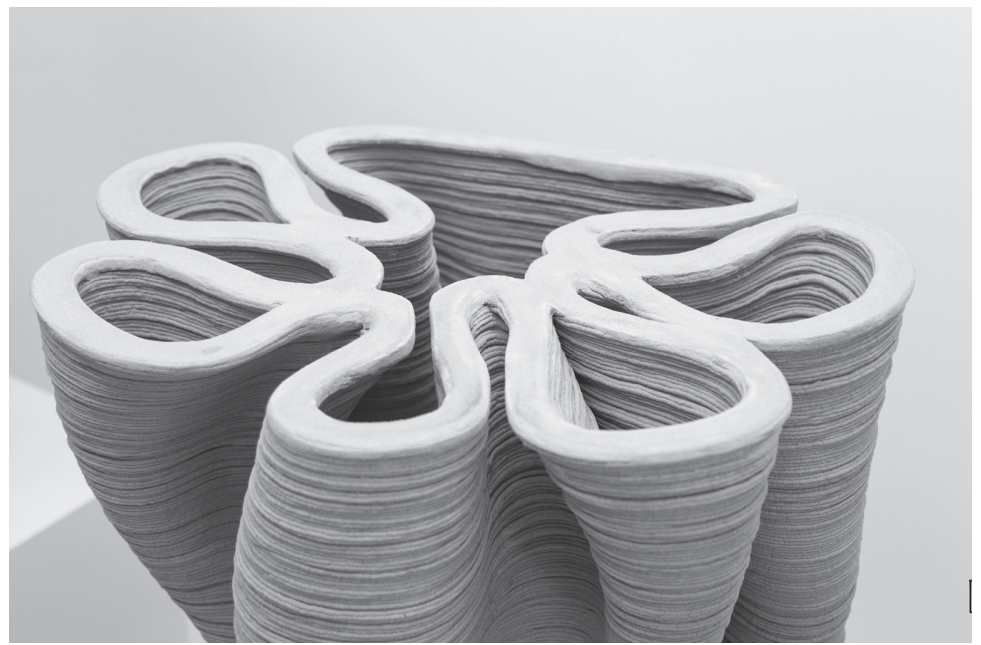

Es fundamental recordar que en esta utopía las nuevas ciudades deben ser capaces de autogestionarse, de ser autosuficientes y de garantizar su mantenimiento directo. La idea de una ciudad dividida en sectores es la que debe prevalecer. Los sectores mantendrán su perfil vertical, pero tendrán un crecimiento subterráneo para cumplir con un número cada vez más grande (y nuevo) de demandas de los consumidores, como mercados y estacionamientos subterráneos para automóviles autónomos. Hoy, en Chandigarh, se emiten multas electrónicas, denominadas e-challans, para los ciudadanos que infringen las reglas de tránsito. Así, mediante el análisis informático, la policía administra mejor el tránsito en las calles desviando el tránsito según la congestión y el servicio de emergencias, que usa la misma información. Hablamos de situaciones actuales: automóviles eléctricos de bajo consumo y sensores que permiten poner multas electrónicas. En poco tiempo tendremos entre nosotros vehículos no tripulados que podrán moverse según las mismas reglas y parámetros. Sin duda, la incorporación de este nuevo tipo de vehículos requerirá una nueva regulación y cambiará el sentido de la movilidad, tal como la conocemos. 


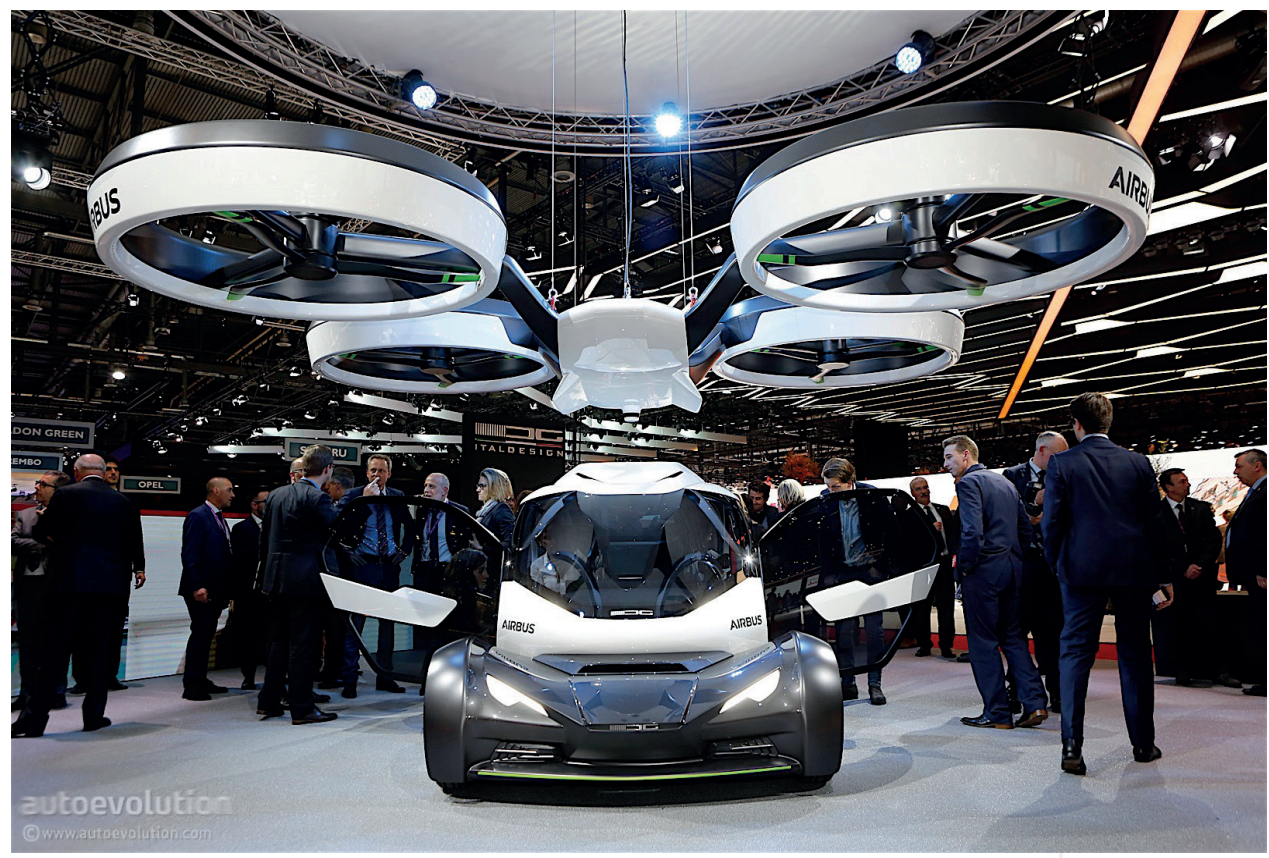

En la actualidad, muchos de nosotros tenemos en casa drones semiprofesionales o profesionales. Gracias a ellos podemos fotografiar y grabar en video situaciones que trascienden nuestras limitaciones físicas. En consecuencia, se ha regulado su uso en lo que respecta a la invasión de privacidad. La tecnología evoluciona, cambia y lo hace rápidamente en un corto tiempo. La siguiente tecnología de drones se manejará con inteligencia artificial y, además de reducir considerablemente la cantidad de riesgos actuales y de impacto, será autónomo y útil para varios fines. Hay varias empresas, como Pilot AI Labs e Iris Automation, que vienen realizando estudios y pruebas en drones; por ejemplo, están desarrollando una visión computarizada para reducir los riesgos de impacto. Su tecnología permite detectar y rastrear objetos incluso en ambientes oscuros. Neurala ha desarrollado un software de redes neuronales de aprendizaje profundo que fabrica productos inteligentes, como drones, automóviles o robots autónomos y útiles.

Estos cambios influirán en los vehículos de transporte de las empresas de logística, como Amazon, Flipkart o Linio; de las de entrega de alimentos, como Zomato, Swiggy, Wong o Tottus; de las de mensajería, como servicios postales, DHL, FedEx, Glovo o Rappi. Depender de drones conscientes de sí mismos, en la próxima década, y no de vehículos tripulados para estos fines cambiará la capacidad y el enfoque actual de estas grandes empresas de logística. La tecnología de los drones automáticos facilitará el proceso de logística y lo hará más eficiente.

Figura 7.

Automóvil

volador eléctrico

Show automotor de Ginebra

Fuente: https:// www.autoevolution.com/ news/italdesignpopup-conceptrevealed-ingeneva-it-s-anidea-for-a-flyingcar-115986. html\#agal_0 
La inteligencia artificial y los dispositivos conectados también desempeñarán un papel importante: automatizarán las unidades de recolección de residuos cuando la demanda de la ciudad sea alta y atenderán a las personas que necesiten ayuda. No cabe duda de que este será un punto de grandes cambios.

Muchos no pueden usar los esquemas de asistencia social disponibles para las personas locales que están por debajo de la línea de pobreza, como la atención médica subsidiada. Esto se hace posible introduciendo el concepto de "centro de atención al migrante", que sería una solución integral para los migrantes que se trasladan de las periferias al centro. Esto también inculcará la dignidad del trabajo y creará más cuidado del medioambiente, la seguridad y la salud. Asimismo, servirá como un centro de registro de migrantes. Esta información podría servir como base de datos para planificar programas de investigación, políticas y actividades. También sería un centro para administrar programas de beneficios sociales de los gobiernos. El sistema de búsqueda de empleo optimizado por inteligencia artificial del centro de atención al migrante podría proveer miles de oportunidades para la fuerza de trabajo.

El concepto de cadena de bloques se usará no solo en la elaboración de bases de datos de registros de nombres, sino también en el campo de las criptomonedas. En esta utopía no existe el dinero en efectivo.

La inteligencia artificial también traerá cambios para las personas con discapacidad. Las personas que ahora no pueden viajar ni disfrutar de su ciudad por las limitaciones físicas que la misma ciudad les impone. Las máquinas usarán la tecnología de una manera totalmente diferente. Tal vez no recolecten ni procesen la información, pero aprenderán de ello para realizar ciertas tareas de manera flexible. La inteligencia artificial permitirá que las personas se comuniquen sin que el lenguaje sea un impedimento y que se desplacen por diferentes ciudades sin que la movilidad reducida sea un impedimento. Lo más importante es que, en esta utopía, todos pueden comunicarse y movilizarse desde donde estén. Los códigos de movilidad y comunicación serán los mismos, porque la inteligencia artificial borrará las diferencias.

Dijimos en un comienzo que la tecnología también trae cambios sociales porque se puede imaginar la interacción social a través de ella. Esto será posible porque cada sector de estas nuevas ciudades tendrá espacios físicos para creadores; es decir, espacios donde se agrupen personas con conocimientos y recursos en común, para crear productos.

Es necesario introducir nuevos tipos de comercios y reemplazar los obsoletos, según las posibilidades de empleo y la demanda del mercado. El desarrollo planificado de la fuerza de trabajo y de sus habilidades deben ser una prioridad para cada sector y esto permitirá el futuro desarrollo de las ciudades, que debe ser integral. Por lo tanto, es necesario usar de manera óptima el espacio disponible para esta importante causa social y guiar las estrategias de desarrollo de las habilidades. 
Cabe mencionar que estos espacios ya existen, pero casi nadie los conoce. En unos años, recibir conocimiento y establecer relaciones sociales en un espacio para creadores será tan común como ir al supermercado. El espacio para creadores mejorará el aprendizaje y las relaciones sociales, independientemente del sexo, la edad, la discapacidad o el lugar de origen. La ubicación física tampoco será un impedimento, porque, gracias al desarrollo de la tecnología de hologramas en tiempo real, se puede trabajar con personas que están en otras regiones.

Uno de los puntos de encuentro entre nuestras ciudades es el legado cultural que subyace bajo el desarrollo actual de nuestras ciudades. Este artículo no tiene por finalidad discutir sobre qué hacer con los restos arqueológicos, si enterrarlos, como se hizo en Chandigarh, o dejarlos a su suerte, como sucede en Lima. Lo que sí es materia de análisis es cómo preservar esta información de generación en generación. Creemos que en este punto el uso de la tecnología es fundamental.

Hoy la impresión en 3D es una gran aliada. Lima hizo posible la reconstrucción hipotética de toda una huaca. Este objeto se exhibió en la Bienal de Venecia 2018 y tanto locales como turistas pudieron conocer sobre el legado cultural de Lima y su estado actual. Esta podría ser una buena manera de perpetuar el legado cultural de cada región y transmitir el conocimiento a las futuras generaciones. De manera similar, en el proceso, muchos otros aprenden a usar la tecnología a su favor. Tal vez en unos años conozcamos otras culturas y otros países usando hologramas cambiantes en espacios públicos. Son espacios que ponen la cultura y el aprendizaje a disposición de todos mediante la interacción con otras personas y con la tecnología.

Figura 8.

Huaca

Pachacámac

impresión 3D.

Pabellón Perú,

Bienal de

Venecia 2018

Fuente: https:// www.archdaily. pe/pe/895129/ en-reserva-pabellon-de-peruen-la-bienal-devenecia-2018

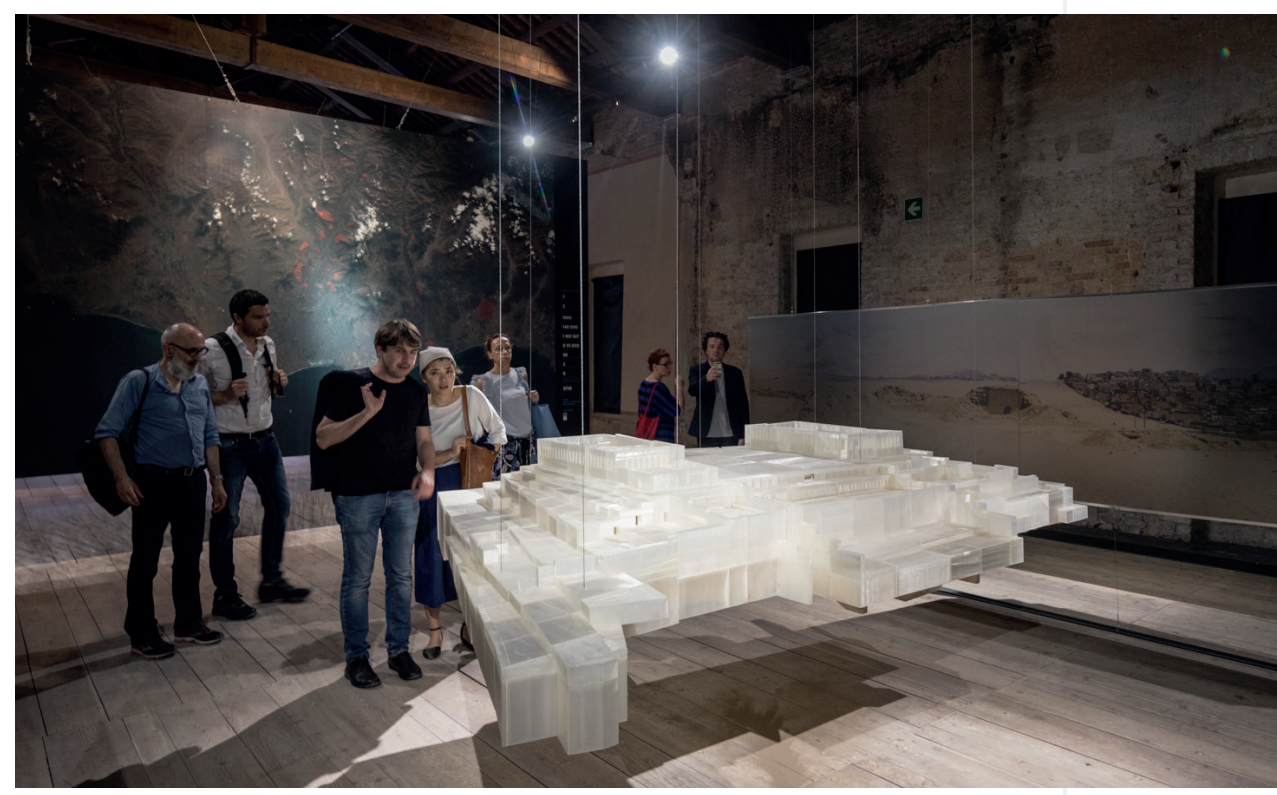


"Los arquitectos deben poder desempeñarse tanto en la interacción con el pasado como con el análisis sobre el futuro. Toda vez que el futuro del pasado depende de él" (Mehrotra, 2017).

\section{CONCLUSIONES}

Se presentan las siguientes ideas para su reflexión y debate:

1. Los seres humanos evolucionarán con el uso de la tecnología, entendiendo el potencial del desarrollo físico y social que esta les permita.

2. Las personas serán las generadoras de la vida social y las máquinas se convertirán en un sistema de apoyo a su alrededor.

3. La inteligencia artificial ofrecerá toda una variedad de alternativas para los seres humanos, pero serán estos quienes tengan la última palabra.

4. Habrá más opciones de fuentes de energía que sean más eficientes y respetuosas con el medioambiente.

5. En el sector de la salud habrá más sistemas de asistencia con inteligencia artificial.

6. En el sector financiero, la cadena de bloques abrirá nuevas rutas en la conducción de transacciones financieras diarias.

No hay una única visión de cómo serán las ciudades en el futuro.

Las ciudades, como las conocemos hoy, no son las mismas en las que vivieron nuestros ancestros. El uso y el desarrollo avanzado de la tecnología, pero, sobre todo, su incorporación en nuestro día a día, generarán más oportunidades y diversidad en la manera en que nos relacionamos social y físicamente. Lo interesante es la superposición de nuevas tecnologías que darán paso a un paisaje urbano nuevo y utópico en el que las reglas de desarrollo ya no serán de naturaleza física, sino de avances tecnológicos y de su aplicación.

Creemos que el uso de la tecnología puede ayudar a reconstruir, pero también a modelar un nuevo paisaje urbano y a proporcionarnos una identidad y memoria colectiva que deje de ser local para ser global. Una identidad que no pertenece a una sola región, sino a todas. 


\section{REFERENCIAS}

Blackmore, S. (1999). The Meme Machine. New York: Oxford University Press.

Bonilla Di Tolla, E. (2015). Lima, ciudad renacentista. Contratexto, (23), pp. 113-125.

Canziani, J. (2013). Territorio, monumentos prehispanicos y paisaje. En J. H. Mazuré, Lima: espacio público, arte y ciudad, p. 78. Lima: Pontificia Universidad Católica del Perú.

Castells, M. y Muñoz, B. F. (2006). La sociedad red: una visión global. Madrid: Alianza Editorial.

Corner, J. (1999) Recovering Landscape: Essays in Contemporary Landscape Architecture. Nueva York: Princeton Architectural Press.

Crousse, J. P. (2017). Agujeros negros urbanos. Desarrollo y patrimonio en la metrópolis de Lima. En J. P. Crousse, Urban Black Holes. Development and heritage in the metropolis of Lima (p. 10-11). Lima: Patronato Cultural del Perú.

Davis, D. (2017). Preface. En J. P. Crousse, Urban Black Holes. Development and heritage in the metropolis of Lima (p. 19). Lima: Patronato Cultural del Perú.

Evans-Cowley, J. (February 2019). The Technology Enhanced City. Planetizen. Recuperado de https://www.planetizen.com/node/63336/

Gehl, J. (2014) Ciudades para la gente. (Primera edición en español). Buenos Aires: Ediciones Infinito.

Ito, T. (2011). Tarzans in the Media Forest. London: Architectural Association.

Live, T. T. (February 2019). 5 Interesting Facts You Probably Didn't Know About Chandigarh. Tricity Live. Recuperado de http://tricitylive.in/5-interesting -facts-you-probably-didnt-know-about-chandigarh/

Ludeña, W. (2013) Lima y espacios públicos. Perfiles y estadística integrada 2010. Lima: Pontificia Universidad Católica del Perú. Oficina de Publicaciones.

McCormack-Noonan, J. (May 2019). Megaciudades del futuro. Viva Mundo. Recuperado de https://viva-mundo.com/es/noticia/post/megaciudadesfuturo?fbclid=IwAR0bLTe83z_QUpxtmmX91j5yAxmNchVV0H063Vv1rFHu EqzfRw5ZMTIteqY

Planning, I. F. (March 2019). The Role of Technology in Urban Development. Recuperado de https://www.ifhp.org/role-technology-urban-development

Ratnagar, S. (abril del 2019). Why have Western and Indian experts studied the Harappan civilisation? Harappa.com. Recuperado de https://www. harappa.com/answers/why-have-western-and-indian-experts-studiedharappan-civilisation

Realizing Society 5.0. (abril del 2019). Japan.go. Recuperado de https://www.japan. go.jp/abenomics/_userdata/abenomics/pdf/society_5.0.pdf 
Sassen, S. (1999). La ciudad global. Nueva York, Londres, Tokio. Buenos Aires: EUDeBA.

Staines, N. (febrero del 2019). Smarter Cities: Using Technology to Improve City Living. Recuperado de https://www.r2integrated.com/r2insights/ smarter-cities-using-technology-to-improve-city-living

Thuroczy, M. (febrero del 2019). City of Chandigarh. Architectuul. Recuperado de http://architectuul.com/architecture/city-of-chandigarh

Traynor, V. (marzo del 2019). The future of: Aged Care. Recuperado de https:/l magazine.uow.edu.au/research/newsletter/2018/UOW251342.html

Verhulst, S. (junio del 2019). Designing for Cities: Technology and the Urban Experience. The Livign Library. Recuperado de https://thelivinglib.org/ designing-for-cities-technology-and-the-urban-experience/

Zukin, S. (2010) Naked City: The Death and Life of Authentic Urban Places. Nueva York: Oxford University Press. 